\title{
Demonstrating the Decline in Coronary Heart Disease Mortality in the West Delhi, India between 2014 to 2017
}

\author{
KUMAR ANKUSH
}

A central University JMI, Biosciences, Delhi, India.

\section{Abstract}

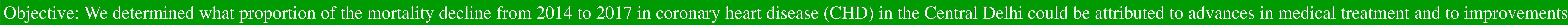

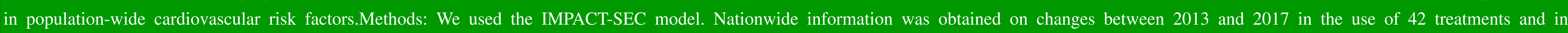

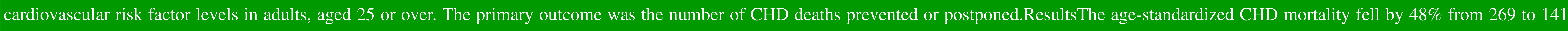

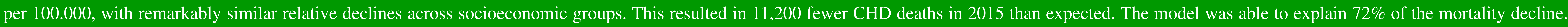

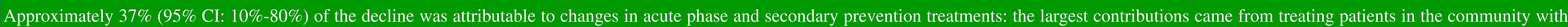

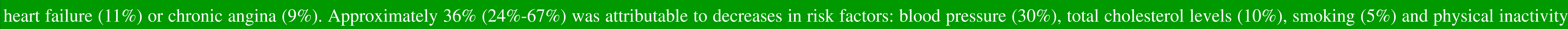

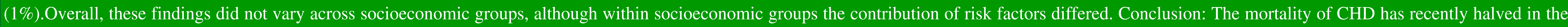

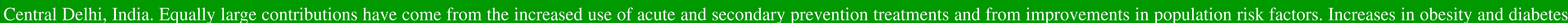
represent a major challenge for future prevention policies.

\section{Background}

$>$ Coronary heart disease (CHD) remains the leading cause of death worldwide and is a major contributor to chronic disease morbidity. Since the 1970s, CHD mortality has fallen dramatically in Western societies. The IMPACT model has been developed to estimate the contribution of changes in uptakes of evidencebased treatments and nationwide changes in cardiovascular risk factors to the changes in CHD mortality. The model has been applied in more than 20 countries Results vary by country, with the contribution from treatments ranging from 25 $50 \%$ and risk factor changes explaining $50-75 \%$. These differences between countries can mostly be explained by the precise time period chosen, and the scale of change in major CHD risk factors.

$>$ We therefore aimed to determine what proportion of the recent decline (from 2014 to 2017) in CHD mortality could be attributed to advancements in medical treatment and to nationwide time trends in CHD risk factors, particularly in socioeconomic subgroups of the population

\section{Materials and Methods}

The Indian population aged 25 years and over between 2014 and 2017 was evaluated using the IMPACT-SEC model. This model integrates nationwide data at two time points to explain an observed change in mortality. The IMPACT model was developed to model CHD mortality trends and incorporates time trends in uptake of evidencebased acute phase and secondary prevention treatments, in addition to time trends in major CHD risk factors and (2) estimate the relative change in CHD mortality associated with each of these items. The IMPACT model is validated and applied i several countries(II)

Data sources

Data used are described in detail in the S1 Appendix. In short, data on the age, sex an socioeconomic distribution of the Indian population and on specific CHD death counts. Indian inhabitants were divided in three socioeconomic groups: lowest $(20 \%$ most deprived persons within age-sex-stratum), medium (60\% of age-sex-stratum) an highest (20\% most affluent persons within age-sex-stratum). Socioeconomic circumstances were based on an area-level socioeconomic indicator constructed by the Indian Institute for Social Research in 2012-2016. The national Indian hospital discharge register was used to determine the number of eligible patients and their 1-year mortality rate. Information on drug use came from the PHARMO Database Network linking pharmacy and hospitalization records of over 2.3 million subjects. Data on drug use in the community came from the general practitioners (GP). Data of major cardiovascular risk factors (blood pressure, cholesterol, body mass index (BMI), diabetes, smoking, physical inactivity) came from the Doetinchem/MORGEN (RIVM) cohort for those aged up to 65 years cohort for those aged 65 years and over. Smoking information came from annual nationwide surveys on smoking habits (STIVORO) an diabetes information from the GP register.

Sensitivity analyses

For each model parameter, minimum and maximum plausible values were assigned using the $95 \%$ confidence intervals (from the source documentation); if these were unavailable, these limits were defined as $20 \%$ above and below the best estimate. The minimum and maximum plausible values were introduced into the model, generating 